Aus der Klinik für Klinik für Hals-, Nasen-, Ohrenheilkunde der Medizinischen Fakultät Charité - Universitätsmedizin Berlin

\title{
DISSERTATION
}

\section{Meta-analysis of subjective complaints of vertigo and vestibular tests after Cochlear Implantation}

\author{
zur Erlangung des akademischen Grades \\ Doctor medicinae (Dr. med.) \\ vorgelegt der Medizinischen Fakultät \\ Charité - Universitätsmedizin Berlin \\ von
}

\section{Toni Hänsel}

aus Stendal

Datum der Promotion: 07.12.2018 

Inhaltsverzeichnis

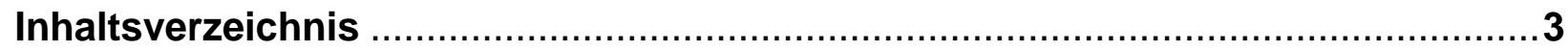

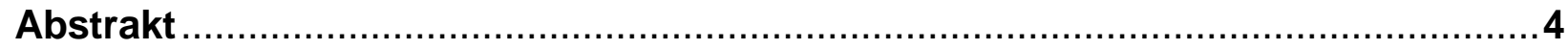

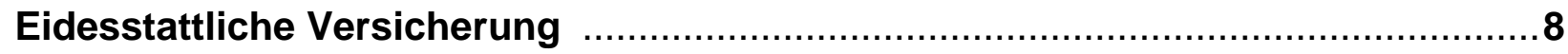

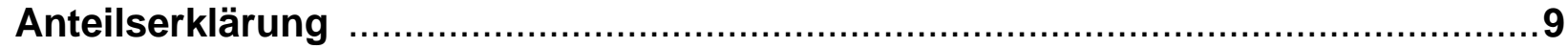

Auszug aus der Journal Summary List (ISI Web of Knowledge ${ }^{\mathrm{SM}}$ ) ....................12

Publikation „Meta-Analysis of subjective complaints of vertigo and vestibular tests after Cochlear Implantation"

\section{Supplementäre Daten}

Supplemental figure 1: Quality of vertigo before / after cochlear implantation..... .28

Supplemental figure 2: Funnel plot - Nystagmography ...................................29

Supplemental figure 3: Funnel plot - VEMP ................................................ 30

Supplemental figure 4: Funnel plot - Vertigo............................................... 31

Supplemental figure 5: Risk of bias analysis - Nystagmography .........................32

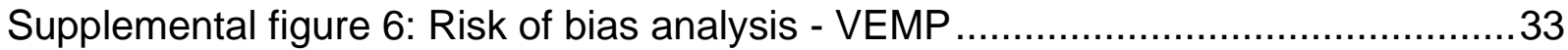

Supplemental figure 7: Risk of bias analysis - Vertigo.....................................34

Supplemental figure 8: Cut off values hyporeflexia in Nystagmography...................35

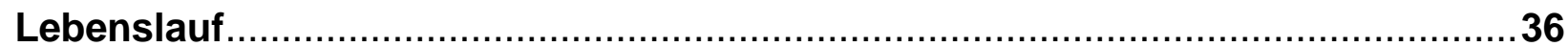

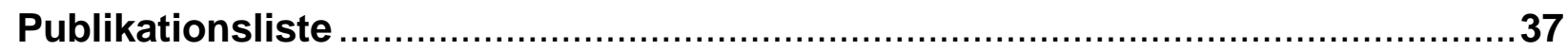

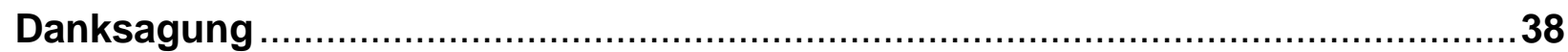




\section{Abstrakt}

Hintergrund. Postoperativ auftretender Schwindel ist eine häufige Komplikation nach Versorgung mit einem Cochlea Implantat $(\mathrm{Cl})$. Die Gründe hierfür sind vielseitig, so kann das $\mathrm{Cl}$ die physiologische Funktion des Corti-Organs und der Labyrinth-Strukturen schädigen. In der Literatur finden sich stark divergierende Häufigkeitsangaben, so setzt sich die vorliegende Studie zum Ziel, sowohl subjektive Beschwerden als auch etablierte Diagostikmethoden zu untersuchen.

Methoden. Es wurde eine systematische Literaturrecherche nach Vorgabe des PRISMA-Statements (Preferred Reporting Items for Systematic Reviews and MetaAnalyses) in PubMed, Cochrane Register und EMBASE durchgeführt. Mit den Suchkriterien "cochlear implant dizziness OR cochlear implant vertigo OR cochlear implant vestibular" wurden 116 aus 2084 Studien ausgewählt, welche über perioperativ aufgetretenen Schwindel, Nystagmographie oder Vestibulär Evozierte Myogene Potentiale (VEMP) berichteten. Drei Metaanalysen wurden aus 46 geeigneten Studien erstellt, welche prä- und postoperative Daten publizierten. Daraus konnte das Odds Ratio neu aufgetretenen Schwindels, sowie eine etwaige Funktionseingeschränkung der Gleichgewichtsrezeptoren gemessen werden. Mit gepoolten Daten aus 95 Studien wurde die Häufigkeit der subjektiven Symptome berechnet und zusätzlich nach Durchschnittsalter unterteilt.

Ergebnisse. Die Metaanalysen demonstrieren eine signifikant erhöhte Chance nach Versorgung mit einem Cochlea Implantat neu aufgetretenen Schwindel zu erleiden. Des Weiteren war eine signifikante, vestibuläre Funktionseinschränkung - ermittelt mit VEMP und Nystagmographie - nachweisbar. In den gepoolten Daten trat Schwindel postoperativ insgesamt zu 9,3\% auf, wobei ein kontinuierlicher Anstieg mit dem Implantationsalter zu verzeichen war. In einer Untergruppe der Studien kam es bei $17,4 \%$ zu neu aufgetretenem Schwindel. 7,2\% der Patienten beschrieben persitierende Beschwerden vor und nach Implantation, während $11,6 \%$ eine Veränderung der Schwindelqualität angaben und 7,7\% lediglich präoperativ Schwindelsymptome beklagten. Ein Vergleich der OP-Zugänge über die Rundfenstermenbran und klassischer Cochleostomie ergab ein signifikant erhöhtes Risiko für postoperativen Schwindels nach Cochleostomie. Eine über VEMP und Nystagmographie festgestellte vestibuläre Funktionseinschränkung zeigte keinen signifikanten Unterschied der beiden OP-Zugänge. 
Schlussfolgerung. Die Versorgung mit einem Cochlea Implantat hat erheblichen Einfluss auf das subjektive Schwindelempfinden und die Funktion der vestibulären Rezeptoren. Das Patientenalter zum Zeitpunkt der Implantation spielt dabei eine wesentliche Rolle; junge $\mathrm{Cl}$-Kandidaten scheinen eine Operation im besseren Maße zu kompensieren als ältere. Die chirurgische Implantationstechnik (Rundfenster oder Cochleostomie) beeinflusst das Ergebnis und solte in weiteren Studien weitergehend untersucht werden. Eine umfangreiche perioperative Diagnostik ist die Voraussetzung um subjektive Symptome mit einer messbaren Rezeptorschädigung zu korrelieren. 


\section{Abstract}

Objective: Among the complaints after cochlear implantation, vertigo is a minor but common complication. There are several explanations, since the cochlea implant $(\mathrm{Cl})$ might harm the physiology and anatomy of the organ of Corti and the labyrinth. The symptoms may result from the surgery, from the $\mathrm{Cl}$ itself, or from changes in the vestibular organ, but also be an effect of the central nervous system. The incidence rates vary vastly in literature; this study therefore intends to clarify both the perioperative vestibular sensations and the diagnostic methods.

Methods: A systematic literature search in line with the Preferred Reporting Items for Systematic Reviews and Meta-Analyses statement was carried out. PubMed, Cochrane Central Register and EMBASE were the sources used. With the search terms "cochlear implant dizziness OR cochlear implant vertigo OR cochlear implant vestibular", we identified 116 studies that reported either subjective complaints, results of nystagmography or results of Vestibular Evoked Myogenic Potentials (VEMP). 46 studies were suitable to be included in the three meta-analyses, which show the odds ratio of postoperative results in VEMP, nystagmography and new onset of vestibular symptoms.

Results: The meta-analyses demonstrate a significantly increased risk to suffer vertigo after cochlear implantation. Furthermore, they show a significant decrease of reflectivity in nystagmography and disappeared VEMPs. The pooled data reveal an average occurrence of vertigo in $9.3 \%$ of the patients after surgery. Several studies reported a new onset of vertigo, which applies to $17.4 \%$ of $\mathrm{Cl}$ user. Persisting vertigo was the case for $7.2 \%$. Moreover, $11.6 \%$ of the patients sensed a change in vertigo quality, while $7.7 \%$ reported vertigo only before implantation. In addition, we observed a continuous increase of postoperative vertigo along with rising age at implantation. In a subgroup analysis of the two surgical approaches - round window and classic cochleostomy -, a higher occurrence of postoperative vertigo was described for the latter. The effects on vestibular function measured by nystagmography and VEMP appeared to be alike.

Conclusion: $\mathrm{A}$ high rate of patients treated with a $\mathrm{Cl}$ suffers vertigo symptoms after surgery. The physiologic function of vestibular receptors is significantly impaired. The round window and the cochleostomy approaches may affect the rate of postoperative 
vertigo incidence. Further studies are needed to validate these findings. The risk of subjective complaints after implantation depends to a high degree on the age at time of surgery, which might be related to a better compensation capacity in younger patients. 


\section{Eidesstattliche Versicherung}

„Ich, Toni Hänsel, versichere an Eides statt durch meine eigenhändige Unterschrift, dass ich die vorgelegte Dissertation mit dem Thema: „Meta-analysis of subjective complaints of vertigo and vestibular tests after Cochlear Implantation" selbstständig und ohne nicht offengelegte Hilfe Dritter verfasst und keine anderen als die angegebenen Quellen und Hilfsmittel genutzt habe.

Alle Stellen, die wörtlich oder dem Sinne nach auf Publikationen oder Vorträgen anderer Autoren beruhen, sind als solche in korrekter Zitierung (siehe „Uniform Requirements for Manuscripts (URM)" des ICMJE -www.icmje.org) kenntlich gemacht. Die Abschnitte zu Methodik (insbesondere praktische Arbeiten, Laborbestimmungen, statistische Aufarbeitung) und Resultaten (insbesondere Abbildungen, Graphiken und Tabellen) entsprechen den URM (s.o) und werden von mir verantwortet.

Mein Anteil an der ausgewählten Publikation entspricht dem, der in der untenstehenden gemeinsamen Erklärung mit der Betreuerin, angegeben ist.

Die Bedeutung dieser eidesstattlichen Versicherung und die strafrechtlichen Folgen einer unwahren eidesstattlichen Versicherung (\$156,161 des Strafgesetzbuches) sind mir bekannt und bewusst." 


\section{Anteilserklärung an der erfolgten Publikation}

Hänsel T, Gauger U, Bernhard N, Behzadi N, Romo Ventura ME, Hofmann V, Olze H, Knopke S, Todt I, Coordes A. Meta-analysis of subjective complaints of vertigo and vestibular tests after Cochlear Implantation. Laryngoscope 2018

Impact Factor: 2.471

Als Erstautor der oben genannten Publikation „Meta-analysis of subjective complaints of vertigo and vestibular tests after Cochlear Implantation“ führte Toni Hänsel nach Festlegung der Suchterme, cochlear implant dizziness OR cochlear implant vertigo OR cochlear implant vestibular' eine Literaturrecherche in den Suchmaschinen PubMed (US National Library of Medicine), Cochrane Central Register of Controlled Trials und in EMBASE (Excerpta Medica dataBASE von Elsevier) durch. Das PRISMA Statement (Preferred Reporting Items for Systematic Reviews and Meta-Analyses) diente als Richtlinie zur Durchführung der geplanten systematischen Übersichtsarbeit. Nach Entfernung doppelter Einträge unter den 2084 Treffern, selektierte er anhand der Abstracts, welche Studien entweder eine Aussage zu Schwindel vor oder nach einer Cochlea Implantation trafen, oder über Untersuchungsmethoden zur Objektivierung der subjektiven Beschwerden berichteten. Er versicherte sich, dass keine weitere Arbeitsgruppe bereits parallel zu diesem Thema forschte und registrierte die laufende systematischen Übersichtsarbeit in der internationalen Datenbank PROSPERO (CRD42017060332). Von einem weiteren Co-Autor wurde eine unabhängige Selektion an den selben Treffern durchgeführt, zusammen mit ersterer verglichen und Diskrepanzen diskutiert.

Im Anschluss wurden tabellarisch verfügbare Daten aus den Volltexten von 155 Studien extrahiert und kontinuierlich evaluiert, welche Aspekte einen Vergleich zulassen. Zu den ermittelten deskriptiven Daten zählte: Land, Institut, Studien Design, Zeitraum, Patientenanzahl, Durchschnittsalter, Altersspanne, Firma des Cochlea Implantats, Modell-Typ, Ätiologie des Hörverlusts und Häufigkeit perioperativ aufgetretenen Schwindels. Ergebnisse folgender Untersuchungsmethoden wurden extrahiert: Video/Elektronystagmographie, zervikal/okulär Vestibulär Evozierte Myogene Potentiale (VEMP), Kopfimpulstest, Computerized Dynamic Posturography (CDP), haptische subjektive Vertikale (HSV), Drehstuhl-Untersuchung, Bruininks-Oseretsky Test of Motor 
Proficiency (BOT-2), Dizziness Handicap Inventory (DHI) und Activities-specific Balance Confidence $(A B C)$ scale.

Die Grenzwerte für die Definition einer Hyporeflektivität bei der Interpretation der Ergebnisse der Nystagmographie variieren zwischen mehreren Institutionen, sodass Toni Hänsel eine tabellarische Übersicht erstellte.

Entsprechend der vorhandenen Daten eigneten sich einige Kategorien für eine weiterführende Analyse. Die Studien wurden von Toni Hänsel ein weiteres Mal hinsichtlich der OP-Methode untersucht, um eine Korrelation mit der vestibulären Funktion oder den subjektiven Beschwerden darzulegen. Des Weiteren berichteten mehrere Autoren über verschiedene, perioperativ auftretende Schwindelqualitäten, sodass eine entsprechende Übersicht erstellt werden konnte.

Toni Hänsel überprüfte in einem weiteren Schritt jene Institute, die mehrere Untersuchungen an den selben Patientenpopulationen durchführten und schloss ältere Veröffentlichungen mit sich überschneidenden Ergebnissen vom Vergleich aus.

Aus den gepoolten Daten des perioperativen Schwindels konnten sowohl Durchschnittswerte für insgesamt prä- oder postoperativ auftretenden Schwindel berechnet, als auch postoperativ neu aufgetretener und verschwundener Schwindel ermittelt werden. Des Weiteren erstellte Toni Hänsel eine nach dem Durchschnittsalter unterteilte Häufigkeitsübersicht des postoperativ auftretenden Schwindels.

Für die Metaanalyse wählte er die Publikationen aus, welche ausreichend Informationen für das Ausfüllen einer Vierfeldertafel boten, um das Odds Ratio für nach Cochlea Implantation neu aufgetretenen Schwindel, sowie neu ermittelte Reflektivitätsminderung in Nystagmographie und VEMPs zu berechnen. Mit der freien Programmiersprache $\mathrm{R}$ erstellte er die Metaanalyse mit den graphischen Darstellungen der Ergebnisse als Forest und Funnel Plot. Um die Robustheit der Ergebnisse zu überprüfen, führte er Subanalysen durch, die im Rahmen der Sensitivitätsanalyse jeweils die neusten Studien mit dem Gesamtergebnis verglichen. Um einen Einfluss der Insertionstechnik der Cochlea Elektrode auf die vestibuläre Funktion und subjektiven Symptome nachzuweisen, wurden sechs weitere Sub-Metaanalysen erstellt.

Zur Bewertung der methodischen Qualität der für die Metaanalysen eingeschlossenen Studien führte Toni Hänsel parallel mit einem Co-Autor eine Risk-of-Bias Analyse mit 
dem QUADAS-2-Tool durch. Nach einem Vergleich der Ergebnisse wurden unterschiedliche Bewertungen diskutiert. Das Risiko des systematischen Fehlers wurde anschließend von inm visualisiert.

Aus den durchgeführten Analysen fertigte Toni Hänsel ein Manuskript mit folgender Gliederung an: Abstract, Introduction, Methods (Search Strategy and Inclusion Criteria, Data Extraction, Statistical Analysis), Results (Description of the Included Studies, Perioperative Vertigo, Vertigo Quality, Age and Vertigo, Meta-Analyses, Subgroup Analysis, Sensitivity Analysis, Publication Bias And Risk Of Bias Assessment), Discussion und Conclusions. Das Manuskript wurde mit den Co-Autoren diskutiert und bei dem Top Journal ,The Laryngoscope` veröffentlicht.

Datum, Stempel, Unterschrift der betreuenden Hochschullehrerin Unterschrift des Doktoranden 
Journal Data Filtered By: Selected JCR Year: 2016 Selected Editions: SCIE,SSCI Selected Categories: "OTORHINOLARYNGOLOGY" Selected Category

Scheme: WoS

Gesamtanzahl: 42 Journale

\begin{tabular}{|c|c|c|c|c|}
\hline Rank & Full Journal Title & Total Cites & $\begin{array}{l}\text { Journal Impact } \\
\text { Factor }\end{array}$ & Eigenfactor Score \\
\hline 1 & $\begin{array}{r}\text { HEAD AND NECK- } \\
\text { JOURNAL FOR THE } \\
\text { SCIENCES AND } \\
\text { SPECIALTIES OF THE } \\
\text { HEAD AND NECK }\end{array}$ & 11,063 & 3.376 & 0.018580 \\
\hline 2 & Trends in Hearing & 139 & 3.024 & 0.000660 \\
\hline 3 & $\begin{array}{r}\text { JAMA Otolaryngology-Head } \\
\& \text { Neck Surgery }\end{array}$ & 1,422 & 2.951 & 0.006550 \\
\hline 4 & HEARING RESEARCH & 8,833 & 2.906 & 0.011270 \\
\hline 5 & EAR AND HEARING & 4,791 & 2.842 & 0.007950 \\
\hline 6 & $\begin{array}{r}\text { CLINICAL } \\
\text { OTOLARYNGOLOGY }\end{array}$ & 2,861 & 2.523 & 0.002770 \\
\hline 7 & LARYNGOSCOPE & 21,551 & 2.471 & 0.028110 \\
\hline 8 & $\begin{array}{r}\text { JARO-JOURNAL OF THE } \\
\text { ASSOCIATION FOR } \\
\text { RESEARCH IN } \\
\text { OTOLARYNGOLOGY } \\
\end{array}$ & 2,000 & 2.455 & 0.004750 \\
\hline 9 & RHINOLOGY & 2,463 & 2.350 & 0.003910 \\
\hline 10 & $\begin{array}{r}\text { OTOLARYNGOLOGY-HEAD } \\
\text { AND NECK SURGERY }\end{array}$ & 12,598 & 2.276 & 0.017930 \\
\hline 11 & $\begin{array}{r}\text { International Forum of } \\
\text { Allergy \& Rhinology }\end{array}$ & 1,809 & 2.135 & 0.005600 \\
\hline 12 & DYSPHAGIA & 2,539 & 2.077 & 0.002940 \\
\hline 13 & $\begin{array}{r}\text { OTOLOGY \& } \\
\text { NEUROTOLOGY }\end{array}$ & 6,695 & 2.024 & 0.012190 \\
\hline 14 & $\begin{array}{r}\text { American Journal of } \\
\text { Rhinology \& Allergy }\end{array}$ & 3,528 & 1.955 & 0.004630 \\
\hline 15 & $\begin{array}{r}\text { AUDIOLOGY AND NEURO- } \\
\text { OTOLOGY }\end{array}$ & 1,659 & 1.791 & 0.002780 \\
\hline 16 & $\begin{array}{r}\text { INTERNATIONAL JOURNAL } \\
\text { OF AUDIOLOGY }\end{array}$ & 2,940 & 1.733 & 0.005150 \\
\hline 17 & $\begin{array}{r}\text { EUROPEAN ARCHIVES OF } \\
\text { OTO-RHINO- } \\
\text { LARYNGOLOGY }\end{array}$ & 6,620 & 1.660 & 0.013520 \\
\hline 18 & $\begin{array}{r}\text { Acta Otorhinolaryngologica } \\
\text { Italica }\end{array}$ & 1,232 & 1.530 & 0.001810 \\
\hline 19 & $\begin{array}{r}\text { Current Opinion in } \\
\text { Otolaryngology \& Head and } \\
\text { Neck Surgery }\end{array}$ & 1,852 & 1.513 & 0.003160 \\
\hline 20 & $\begin{array}{r}\text { Journal of Otolaryngology- } \\
\text { Head \& Neck Surgery }\end{array}$ & 1,708 & 1.495 & 0.002050 \\
\hline 21 & $\begin{array}{r}\text { ANNALS OF OTOLOGY } \\
\text { RHINOLOGY AND } \\
\text { LARYNGOLOGY }\end{array}$ & 6,446 & 1.384 & 0.004930 \\
\hline 22 & JOURNAL OF VOICE & 3,573 & 1.381 & 0.004550 \\
\hline 23 & $\begin{array}{r}\text { OTOLARYNGOLOGIC } \\
\text { CLINICS OF NORTH } \\
\text { AMERICA }\end{array}$ & 2,245 & 1.264 & 0.002970 \\
\hline 24 & $\begin{array}{r}\text { Journal of the American } \\
\text { Academy of Audiology }\end{array}$ & 1,893 & 1.208 & 0.002840 \\
\hline 25 & $\begin{array}{r}\text { INTERNATIONAL JOURNAL } \\
\text { OF PEDIATRIC } \\
\text { OTORHINOLARYNGOLOGY }\end{array}$ & 6,811 & 1.159 & 0.010860 \\
\hline
\end{tabular}




\begin{tabular}{|c|c|c|c|c|}
\hline Rank & Full Journal Title & Total Cites & $\begin{array}{l}\text { Journal Impact } \\
\text { Factor }\end{array}$ & Eigenfactor Score \\
\hline 26 & $\begin{array}{r}\text { Clinical and Experimental } \\
\text { Otorhinolaryngology }\end{array}$ & 500 & 1.149 & 0.001220 \\
\hline 27 & AURIS NASUS LARYNX & 1,758 & 1.128 & 0.002760 \\
\hline 28 & $\begin{array}{r}\text { ACTA OTO- } \\
\text { LARYNGOLOGICA }\end{array}$ & 6,429 & 1.116 & 0.004870 \\
\hline 29 & $\begin{array}{r}\text { European Annals of } \\
\text { Otorhinolaryngology-Head } \\
\text { and Neck Diseases }\end{array}$ & 426 & 1.108 & 0.001610 \\
\hline 30 & $\begin{array}{r}\text { American Journal of } \\
\text { Audiology }\end{array}$ & 648 & 1.076 & 0.001690 \\
\hline 31 & $\begin{array}{r}\text { ORL-Journal for Oto-Rhino- } \\
\text { Laryngology Head and Neck } \\
\text { Surgery }\end{array}$ & 1,146 & 1.055 & 0.001090 \\
\hline 32 & $\begin{array}{r}\text { AMERICAN JOURNAL OF } \\
\text { OTOLARYNGOLOGY }\end{array}$ & 2,584 & 1.033 & 0.004130 \\
\hline 33 & $\begin{array}{r}\text { ENT-EAR NOSE \& THROAT } \\
\text { JOURNAL }\end{array}$ & 1,585 & 0.919 & 0.001260 \\
\hline 34 & $\begin{array}{r}\text { JOURNAL OF VESTIBULAR } \\
\text { RESEARCH-EQUILIBRIUM } \\
\& \text { ORIENTATION }\end{array}$ & 816 & 0.900 & 0.001050 \\
\hline 35 & $\begin{array}{r}\text { JOURNAL OF } \\
\text { LARYNGOLOGY AND } \\
\text { OTOLOGY }\end{array}$ & 5,410 & 0.844 & 0.004480 \\
\hline 36 & $\begin{array}{r}\text { Brazilian Journal of } \\
\text { Otorhinolaryngology }\end{array}$ & 1,035 & 0.822 & 0.001730 \\
\hline 36 & $\begin{array}{r}\text { Logopedics Phoniatrics } \\
\text { Vocology }\end{array}$ & 400 & 0.822 & 0.000620 \\
\hline 38 & $\begin{array}{r}\text { LARYNGO-RHINO- } \\
\text { OTOLOGIE }\end{array}$ & 735 & 0.732 & 0.000440 \\
\hline 39 & $\mathrm{HNO}$ & 1,072 & 0.723 & 0.000850 \\
\hline 40 & B-ENT & 321 & 0.578 & 0.000720 \\
\hline 41 & $\begin{array}{r}\text { FOLIA PHONIATRICA ET } \\
\text { LOGOPAEDICA }\end{array}$ & 857 & 0.417 & 0.000880 \\
\hline 42 & $\begin{array}{r}\text { Journal of International } \\
\text { Advanced Otology }\end{array}$ & 127 & 0.392 & 0.000350 \\
\hline
\end{tabular}

Copyright (C) 2017 Thomson Reuters 
Hänsel, T. , Gauger, U. , Bernhard, N. , Behzadi, N. , Romo Ventura, M. E., Hofmann, V. , Olze, H. , Knopke, S. , Todt, I. and Coordes, A. (2018), Meta-analysis of subjective complaints of vertigo and vestibular tests after cochlear implantation. The Laryngoscope. . doi:10.1002/lary.27071

https://doi.org/10.1002/lary.27071 
Mein Lebenslauf wird aus datenschutzrechtlichen Gründen in der elektronischen Version meiner Arbeit nicht veröffentlicht. 


\section{Publikationsliste}

Hänsel T, Gauger U, Bernhard N, Behzadi N, Romo Ventura ME, Hofmann V, Olze H, Knopke S, Todt I, Coordes A. Meta-analysis of subjective complaints of vertigo and vestibular tests after Cochlear Implantation. Laryngoscope 2018

Impact Factor: 2.471 


\section{Danksagung}

Hören und Gleichgewicht sind das zentrale Thema dieser Doktorarbeit. Der Philosoph und Kommunikationstheoretiker Marshall McLuhan schrieb: „Das Ohr bevorzugt keinen bestimmten ,Blickwinkel'. Wir sind von Geräuschen umhüllt, in einem lückenlosen Netz. (...) Geräusche können wir nicht einfach ausblenden. Wir haben keine Ohrenlider." Für einige ist das Hören nicht minder bedeutend als die Sehkraft, vielen wird dies jedoch erst nach dessen Verlust bewusst. Sollten wir nicht in Ungleichgewicht geraten, merken wir nicht, dass wir einen Sinn für die Balance besitzen. Auf diesem Weg möchte ich den Menschen danken, die immer ein offenes Ohr für mich hatten und mich mit beiden Beinen auf dem Boden hielten.

Annekatrin Coordes, ich danke dir für die intensive Betreuung und die vielen gemeinsamen Stunden, die zu einer erfolgreichen Promotion und Publikation der Arbeit führten.

Noé, für die konstruktive Kritik und Korrektur über den Atlantik hinweg,

Eugenia, die mehr Licht in den methodischen Dschungel und in mein Leben brachte,

und meiner Familie, die mich stetig bei meinen Ideen und jeglichen Vorhaben unterstützen.

Euer Toni 